\title{
Density Functional Studies of Nonlinear Elastic Properties for Zinc-Blende Nitrides
}

\author{
M. ŁOPUSZYŃSKI ${ }^{a}$ AND J.A. MAJEWSKI ${ }^{b}$ \\ ${ }^{a}$ Interdisciplinary Centre for Mathematical and Computational Modelling \\ Warsaw University, Pawińskiego 5A, 02-106 Warsaw, Poland \\ ${ }^{b}$ Institute of Theoretical Physics, Faculty of Physics, Warsaw University \\ Hoża 69, 00-681 Warsaw, Poland \\ In this paper we present a method of calculating third-order elastic con- \\ stants $C_{i j k}$ and its application to zinc-blende nitrides AlN, GaN, and InN. \\ Our approach is based on accurate $a b$ initio calculations of both energy and \\ stress as a function of applied strain. Ab initio computations are performed \\ within density functional theory framework. To assess the reliability of the \\ presented method, we compare our theoretical findings for GaAs with ex- \\ perimental results for $C_{i j k}$ available for this material.
}

PACS numbers: 62.20.Dc, 43.25.+y, 62.50.+p

\section{Motivation}

The increased interest in semiconducting nanostructures observed in recent years, brought significant attention to nonlinear effects in elastic $[1,2]$, and piezoelectric properties [3,4] of semiconductors. Going beyond a linear description in such systems is very often necessary for their reliable quantitative modelling and the correct theoretical explanation of the experimental data [2, 4-8]. Below we present ab initio studies of the unknown third-order elastic constants in cubic nitrides. The nitrides constitute a group of materials important from the technological point of view, moreover, the nonlinear effects are of particular importance in these semiconductors $[1,2,9,10]$.

\section{Nonlinear elasticity}

Below we provide a very brief overview of nonlinear theory of elasticity (see e.g. [11-13]). Let us consider the point $\boldsymbol{x}$ which, after applying strain to a crystal, moves to the position $\boldsymbol{x}^{\prime}$. After introducing the Jacobian matrix $\boldsymbol{J}$ :

$$
J_{i j}=\frac{\partial x_{i}^{\prime}}{\partial x_{j}}=\mathbf{1}+\boldsymbol{\epsilon},
$$


we may define the Lagrangian strain

$$
\boldsymbol{\eta}=\frac{1}{2}\left(\boldsymbol{J}^{\mathrm{T}} \boldsymbol{J}-\mathbf{1}\right)=\boldsymbol{\epsilon}+\frac{1}{2} \boldsymbol{\epsilon}^{2}
$$

where we assumed that deformation is rotation free and introduced a linear strain tensor $\boldsymbol{\epsilon}$. The energy per unit mass $E(\boldsymbol{\eta})$ corresponding to the applied strain may be developed in power series with respect to $\boldsymbol{\eta}$. This leads to the expression*

$$
\rho_{0} E(\boldsymbol{\eta})=\frac{1}{2 !} \sum_{i, j=1,6} c_{i j} \eta_{i} \eta_{j}+\frac{1}{3 !} \sum_{i, j, k=1,6} C_{i j k} \eta_{i} \eta_{j} \eta_{k}+\ldots
$$

where $\rho_{0}$ is a density of unstrained crystal. The $c_{i j}$ and $C_{i j k}$ denote here secondand third-order elastic constants, respectively. Another fundamental quantity in the theory of finite deformations is Lagrangian stress

$$
t_{i j}=\rho_{0} \frac{\partial E}{\partial \eta_{i j}}
$$

which can be expressed in terms of linear stress tensor $\boldsymbol{\sigma}$ using the following formula:

$$
\boldsymbol{t}=\operatorname{det}(\boldsymbol{J}) \boldsymbol{J}^{-1} \boldsymbol{\sigma}\left(\boldsymbol{J}^{\mathrm{T}}\right)^{-1}
$$

\section{Computational approach}

For the determination of the third-order elastic constants on the basis of density functional theory (DFT) calculations we used two methods. First we made use of strain-energy formula (Eq. (3)), second we employed strain-stress relations (Eqs. (4) and (5)). The computational procedure was as follows (see also [14]). We considered six sets of deformations parameterized by $\eta: \boldsymbol{\eta}_{A}=$ $(\eta, 0,0,0,0,0), \boldsymbol{\eta}_{B}=(\eta, \eta, 0,0,0,0), \boldsymbol{\eta}_{C}=(\eta, 0,0, \eta, 0,0), \boldsymbol{\eta}_{D}=(\eta, 0,0,0, \eta, 0)$, $\boldsymbol{\eta}_{E}=(\eta, \eta, \eta, 0,0,0), \boldsymbol{\eta}_{F}=(0,0,0, \eta, \eta, \eta)$. For every type of deformation we performed DFT calculations using the following values of parameter $\eta$ :

$$
\eta=-0.080,-0.072, \ldots, 0.080 \text {. }
$$

In each calculation the positions of atoms were optimized and both energy and stress tensors were calculated by means of DFT approach. On the other hand, the energy and stress tensors as a function of $\eta$ were obtained from nonlinear theory of elasticity. After substituting the $\boldsymbol{\eta}_{A}, \boldsymbol{\eta}_{B}, \ldots, \boldsymbol{\eta}_{F}$ to Eq. (3) and Eq. (4), one obtains a polynomial dependence of energy $E$ and Lagrangian stress $\boldsymbol{t}$ on the parameter $\eta$, for each deformation family. By fitting the suitable polynomials to the results obtained from DFT and comparing the fits with prediction of nonlinear elasticity, it is straightforward to determine the values of second- and third-order elastic constants.

${ }^{*}$ In the formula for the energy we employed the so-called Voigt convention to shorten the notation, i.e. the components of the symmetric tensor $\eta_{i j}$ were denoted with a single subscript according to $\eta_{11} \rightarrow \eta_{1}, \eta_{22} \rightarrow \eta_{2}, \eta_{33} \rightarrow \eta_{3}, \eta_{23}=\eta_{32} \rightarrow \eta_{4} / 2, \eta_{13}=\eta_{31} \rightarrow$ $\eta_{5} / 2, \eta_{12}=\eta_{21} \rightarrow \eta_{6} / 2$. 
The DFT calculations have been performed with the VASP code using the PAW method [15]. Generalized gradient approximation (GGA) was used according to method of Perdew et al. (PBE) [16].

\section{Benchmark results - GaAs}

To benchmark our approach we performed the test calculations for GaAs. We compared our results with previous calculations for this material performed within DFT-local density approximation (DFT-LDA) framework [17] and available experimental data [18-20]. The results of comparison are given in Table I. The agreement between the theoretical and the experimental results is reasonable, particularly for the elastic constants of larger magnitude $-C_{111}, C_{112}$, and $C_{155}$. For the $C_{144}, C_{123}, C_{456}$ the differences are larger, however, these quantities are also harder to measure (see the deviations between experimental findings of different groups). These results are illustrated in the form of bar graph in Fig. 1. In our opinion, the main sources of discrepancies between theory and experiment
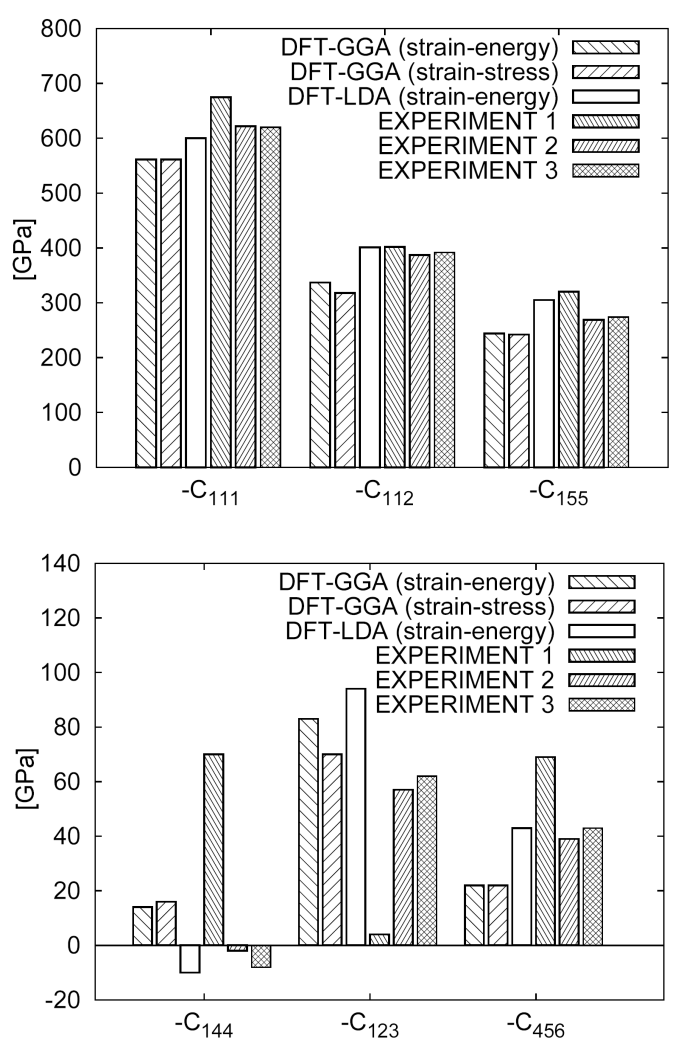

Fig. 1. Comparison of our results for GaAs with previous calculations [17] and experiment $[18-20]$. 
TABLE I

Experimental and theoretical results for GaAs (GPa).

\begin{tabular}{c|c|c|c|c|c|c}
\hline \hline & \multicolumn{2}{|c|}{ Present calculations } & $\begin{array}{c}\text { Previous } \\
\text { calculations }\end{array}$ & \multicolumn{3}{|c}{ Experiment } \\
\cline { 2 - 5 } & energy & stress & $-600^{a}$ & $-675^{b}$ & $-622^{c}$ & $-620^{d}$ \\
\hline$C_{111}$ & -561 & -561 & $-401^{a}$ & $-402^{b}$ & $-387^{c}$ & $-392^{d}$ \\
$C_{112}$ & -337 & -318 & $10^{a}$ & $-70^{b}$ & $2^{c}$ & $8^{d}$ \\
$C_{144}$ & -14 & -16 & $-305^{a}$ & $-320^{b}$ & $-269^{c}$ & $-274^{d}$ \\
$C_{155}$ & -244 & -242 & $-94^{a}$ & $-4^{b}$ & $-57^{c}$ & $-62^{d}$ \\
$C_{123}$ & -83 & -70 & $-43^{a}$ & $-69^{b}$ & $-39^{c}$ & $-43^{d}$ \\
$C_{456}$ & -22 & -22 & & & & \\
\hline${ }^{a}$ Ref. [17] $(\mathrm{LDA})$. & & & & \\
${ }^{b}$ Ref. [18] $(T=298 \mathrm{~K})$. & \\
${ }^{c}$ Ref. [19] $(T=298 \mathrm{~K})$. & \\
${ }^{d}$ Ref. [20] $(T=298 \mathrm{~K})$. &
\end{tabular}

are temperature effects not included in our DFT calculations, the shortcomings of used GGA approximation and measurement uncertainties.

\section{Results for cubic nitrides}

The results for zinc-blende nitrides are displayed in Table II. In addition, our calculations show that nonlinear effects cannot be neglected, when strains larger than approximately $2.5 \%$ are involved. It is also worth noting that for all studied semiconductors and examined range of deformations (i.e., with Lagrangian strains up to $8 \%$ ) including the terms up to third order in energy expansion (Eq. (3)) sufficed to obtain good agreement with DFT results.

TABLE II

Third-order elastic constants for zinc-blende nitrides.

\begin{tabular}{l|r|r|r|r|r|r}
\hline \hline & \multicolumn{3}{|c|}{ Energy } & \multicolumn{3}{c}{ Stress } \\
\cline { 2 - 7 } & AlN & GaN & InN & AlN & GaN & InN \\
\hline$C_{111}$ & -1070 & -1209 & -752 & -1073 & -1213 & -756 \\
$C_{112}$ & -1010 & -905 & -661 & -965 & -867 & -636 \\
$C_{144}$ & 63 & -45 & 16 & 57 & -46 & 13 \\
$C_{155}$ & -751 & -603 & -268 & -757 & -606 & -271 \\
$C_{123}$ & -78 & -294 & -357 & -61 & -253 & -310 \\
$C_{456}$ & -11 & -48 & 14 & -9 & -49 & 15
\end{tabular}




\section{Summary}

We have presented the detailed $a b$ initio study of the third-order elastic constants $C_{i j k}$ for GaAs and zinc-blende nitrides AlN, GaN, InN. Even though third-order effects are very subtle, we showed that it is possible to estimate them by means of density functional theory on the GGA level. The $C_{i j k}$ constitute a useful group of mechanical parameters, their knowledge enables the estimation of other important quantities such as pressure derivatives of second-order elastic constants $c_{i j}^{\prime}$ or mode Grüneisen constants $\gamma(\boldsymbol{q}, \boldsymbol{j})$ (see [14] and references therein). We believe that DFT estimates of third-order elastic moduli can be a useful tool in modelling semiconducting nanostructures, where nonlinear effects often play an important role.

\section{References}

[1] R. Kato, J. Hama, J. Phys., Condens. Matter 6, 7617 (1994).

[2] S.P. Łepkowski, J.A. Majewski, G. Jurczak, Phys. Rev. B 72, 245201 (2005).

[3] K. Shimada, T. Sota, K. Suzuki, H. Okumura, Jpn. J. Appl. Phys. 37, L1421 (1998).

[4] G. Bester, X. Wu, D. Vanderbilt, A. Zunger, Phys. Rev. Lett. 96, 187602 (2006).

[5] M.D. Frogley, J.R. Downes, D.J. Dunstan, Phys. Rev. B 62, 13612 (2000).

[6] S.W. Ellaway, D.A. Faux, J. Appl. Phys. 92, 3027 (2002).

[7] B.S. Ma, X.D. Wang, F.H. Su, Z.L. Fang, K. Ding, Z.C. Niu, G.H. Li, J. Appl. Phys. 95, 933 (2004).

[8] J.W. Luo, S.S. Li, J.B. Xia, L.W. Wang, Phys. Rev. B 71, 245315 (2005).

[9] G. Vaschenko, C.S. Menoni, D. Patel, C.N. Tomé, B. Clausen, N.F. Gardner, J. Sun, W. Götz, H.M. Ng, A.Y. Cho, Phys. Status Solidi B 235, 238 (2003).

[10] S.P. Łepkowski, J.A. Majewski, Phys. Rev. B 74, 035336 (2006).

[11] F. Birch, Phys. Rev. 71, 809 (1947).

[12] F. Murnaghan, Finite Deformation of an Elastic Solid, Wiley, New York 1951.

[13] Y. Hiki, Ann. Rev. Mater. Sci. 11, 51 (1981).

[14] M. Łopuszyński, J.A. Majewski, Phys. Rev. B 76, 045202 (2007).

[15] G. Kresse, D. Joubert, Phys. Rev. B 59, 1758 (1999).

[16] J.P. Perdew, K. Burke, M. Ernzerhof, Phys. Rev. Lett. 77, 3865 (1996).

[17] J. Sörgel, U. Scherz, Eur. Phys. J. B 5, 45 (1998).

[18] J. Drabble, A. Brammer, Solid State Commun. 4, 467 (1966).

[19] H.J. McSkimin, P. Andreatch, J. Appl. Phys. 38, 2610 (1967).

[20] Y. Abe, K. Imai, Jpn. J. Appl. Phys. 25, Suppl. 25-1, 67 (1986). 